REVIEW

\title{
Interstitial lung disease: progress and problems
}

\section{S J Bourke}

Postgrad Med J 2006;82:494-499. doi: 10.1136/pgmi.2006.046417

Interstitial lung disease involves all areas of medicine as it often occurs in patients with comorbidities or as a consequence of systemic diseases and their treatment. Typically the physician is faced with a breathless patient, a diffusely abnormal chest radiograph, and a wide differential diagnosis. Progress has been made in using high resolution computed tomography as the key investigation in characterising the pattern and extent of the disease. Bronchoalveolar lavage is particularly important in excluding infection as a cause of diffuse lung infiltrates. Surgical lung biopsies have led to a new classification system for the range of histopathological patterns of disease that were previously known by the collective term cryptogenic fibrosing alveolitis. Problems persist in deciding when a surgical lung biopsy is clinically justified, in understanding the pathogenesis of these diseases, and in finding more effective treatments.

Correspondence to:

Dr S J Bourke, Department of Respiratory Medicine, Royal Victoria Infirmary, Newcastle upon Tyne NE1 4LP, UK; Stephen.Bourke@ nuth.northy.nhs.uk

Submitted 9 February 2006 Accepted 16 March 2006
$\mathrm{T}$ he terms interstitial lung disease (ILD) and diffuse parenchymal lung disease are imprecise clinical terms for a diverse range of diseases that involve inflammation and fibrosis of the alveoli, distal airways, and septal interstitium of the lungs. ${ }^{1-3}$ Careful clinical investigation aims to move from these imprecise terms to the diagnosis of a specific disease (box 1). ILD involves all areas of medicine as it has to be distinguished from other diseases such as pulmonary oedema, infection, and neoplastic disease, it often occurs as part of a systemic disease or as a reaction to drugs, and it may occur in elderly patients with multiple comorbidities. Advances in the histopathological classification of what was previously known by the collective term cryptogenic fibrosing alveolitis have permitted more precise definition of some disease processes, and this is important in determining prognosis and response to treatment. ${ }^{4-6}$ The lungs may respond to different insults with a similar pattern of inflammation and fibrosis and conversely a single agent, such as amiodarone, may produce a range of reactions within the lung. ${ }^{78}$ Thus a distinction has to be made between the histopathological pattern on lung biopsy and the overall clinical diagnosis, and this frequently entails the integration of clinical, radiological, and histopathological features in a multidisciplinary approach to these complex diseases.

\section{INVESTIGATION OF ILD}

Typically ILD presents with progressive breathlessness, lung crackles, and a diffusely abnormal chest radiograph (fig 1). ${ }^{1-3}$ At presentation the differential diagnosis includes a number of other diseases such as infective pneumonia, pulmonary oedema, and malignancy (for example, lymphangitis carcinomatosa). The overall context of the disease is important and the exclusion of other diagnoses may require further investigations (for example, echocardiography) or observing the response to treatments (for example, antibiotics, diuretics). Lung function tests typically show reduced lung volumes, impaired gas transfer, and hypoxaemia. A reduction in the transfer factor for carbon monoxide and transfer coefficient are characteristic of diseases of the lung parenchyma and its blood supply. These parmaeters are therefore reduced in ILD, but also in emphysema and pulmonary vascular disease. In emphysema the chest radiograph typically shows hyperinflated lungs whereas in ILD the radiograph usually shows reduced lung volumes with reticulonodular infiltrates. Once ILD is suspected the first step is a careful review of the clinical history focusing on occupational history (for example, exposure to asbestos, coal dust), environmental exposures (for example, contact with birds, cigarette smoke), a list of all drugs (for example, amiodarone, methotrexate), and any clues to systemic disease that might involve the lungs. It is essential to be aware of the patient's full medical history and any risk factors for an immunocompromised state as the interpretation of subsequent investigations depends on the clinical context. The presence of eosinophilia, autoantibodies, or avian precipitins may provide useful clues in certain circumstances.

The next step is high resolution computed tomography (HRCT), which gives greater precision than the chest radiograph in diagnosing ILD and in characterising the pattern and extent of the disease. ${ }^{1011}$ The features that may characterise ILD include nodules, thickened septa, reticulation, areas of decreased attenuation, groundglass opacities, honeycombing, and involvement of lymph nodes or pleura in some diseases. Honeycombing with reticular shadowing and secondary bronchial dilatation (traction bronchiectasis) correlates with the histopathological pattern of usual interstitial pneumonia (UIP) and shows established fibrosis with a poor response to corticosteroids ${ }^{11}$ (fig 2). Groundglass opacities away from areas of fibrosis usually is a sign of alveolitis such as is found in

\footnotetext{
Abbreviations: ILD, interstitial lung disease; IPF, idiopathic pulmonary fibrosis; UIP, usual interstitial pneumonia; NSIP, non-specific interstitial pneumonia; DIP, desquamative interstitial pneumonia; COP, cryptogenic organising pneumonia; EAA, extrinsic allergic alveolitis; BAL, bronchoalveolar lavage
} 


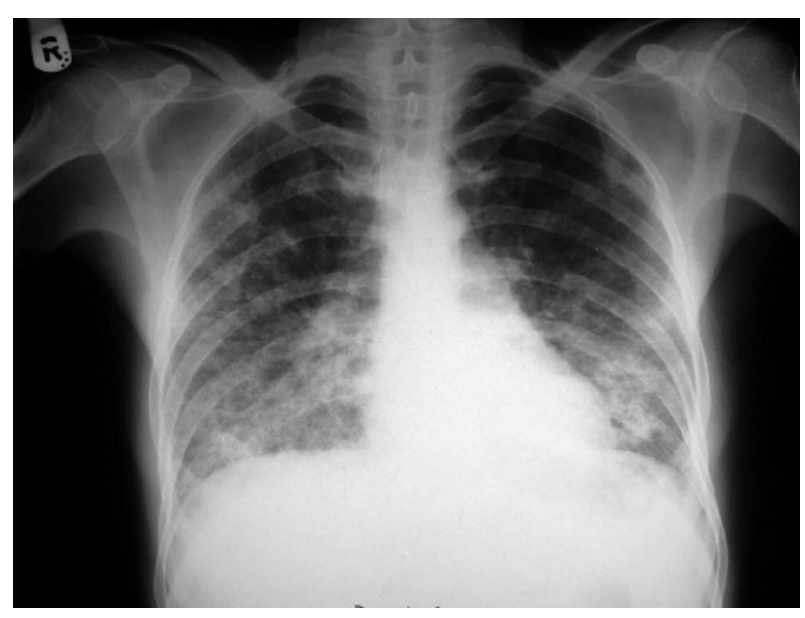

Figure 1 Chest radiograph showing diffuse parenchymal infiltrates attributable to bleomycin pulmonary toxicity.

extrinsic allergic alveolitis (EAA) or non-specific interstitial pneumonia (NSIP), and predict a better response to treatment.

Bronchoalveolar lavage (BAL) is a method of sampling cells and fluid from a large area of the lung tissue by instilling and aspirating saline via a bronchoscope wedged in a segmental bronchus. ${ }^{12}$ It is particularly useful in detecting infection as a cause of diffuse lung infiltrates, especially in patients with human immunodeficiency virus (HIV) infection or in patients receiving chemotherapy for malignancy or immunosuppressive drugs for connective tissue disease, inflammatory bowel disease, or after organ transplantation. An increase in neutrophils and eosinophils in BAL is typically found in idiopathic pulmonary fibrosis (IPF) whereas an increase in lymphocytes is found in sarcoidosis and EAA. Haemosiderin laden macrophages are found in pulmonary haemosiderosis and vasculitis with alveolar haemorrhage (for example, Wegener's granulomatosis) and Langerhans cells can be identified in histiocytosis. ${ }^{13}{ }^{14}$ In pulmonary alveolar proteinosis the BAL fluid has a milky appearance. ${ }^{15}$

Lung biopsy is a key element in the diagnosis of some ILDs such as IPF and in the classification of the idiopathic interstitial pneumonias (box 1), whereas in other diseases such as EAA it is rarely needed. ${ }^{35} 6^{16-18}$ Transbronchial biopsy may be performed via a flexible bronchoscope, at the same time as BAL, and obtains small samples of lung tissue adjacent to bronchi. It is useful, therefore, in centrilobular

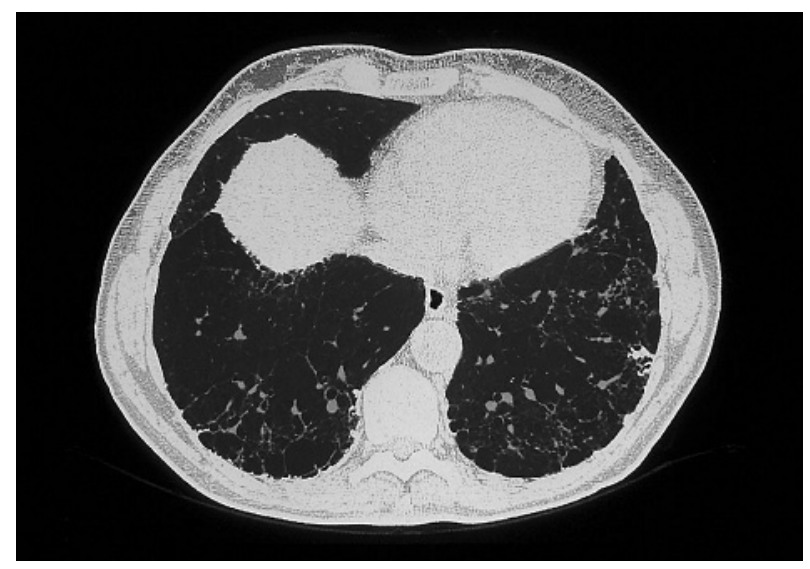

Figure 2 High resolution computed tomography showing subpleural honeycombing in idiopathic pulmonary fibrosis. diseases such as sarcoidosis but it carries a small risk of pneumothorax, haemorrhage, and deteriorating hypoxaemia. ${ }^{19}$ Larger samples from several sites may be obtained by surgical biopsy, usually by video assisted thoracoscopy, and the modern classification of the idiopathic interstitial pneumonias is based on such surgical biopsies. Surgical biopsy requires general anaesthesia with a complication rate of about $10 \%-20 \%$ and a mortality of less than $1 \%$ in the group of patients currently being selected to undergo this procedure. ${ }^{20}$ Some patients are considered unfit for biopsy and the potential benefits of establishing the histopathological pattern of the disease must be judged against the risks of the procedure. Biopsy may not be necessary where the clinical assessment and HRCT features are characteristic of a particular disease. ${ }^{21}$

\section{IDIOPATHIC PULMONARY FIBROSIS (IPF)}

The terms cryptogenic fibrosing alveolitis and IPF were used for many years to incorporate a range of fibrotic lung diseases of unknown cause. There was then puzzlement at the variable clinical course of these syndromes. Progress in our understanding of the different histopathological patterns and the publication of consensus statements have led to some redefinition of these diseases. ${ }^{1-6}$ The overall term idiopathic interstitial pneumonias is used as a general descriptive term and several different histopathological patterns of disease are recognised that vary in their prognosis and response to treatment. The term IPF is now restricted to a specific condition characterised by the histopathological pattern of

\section{Box 1 Classification of interstitial/diffuse parenchymal lung disease}

Idopathic interstitial pneumonias

- Idiopathic pulmonary fibrosis/usual interstitial pneumonia (IPF/UIP)

- Non-specific interstitial pneumonia (NSIP)

- Desquamative interstitial pneumonia (DIP)

- Respiratory bronchiolitis/interstitial lung disease (RB/ ILD)

- Acute interstitial pneumonia (AIP)

- Lymphoid interstitial pneumonia (LIP)

- Cryptogenic organising pneumonia (COP)

Environmental and occupational diseases

- Pneumoconiosis eg asbestosis, silicosis

- Extrinsic allergic alveolitis (EAA), for example, bird fancier's lung

\section{Multisystem diseases}

- Connective tissue diseases, for example, systemic sclerosis,

- Sarcoidosis

- Wegener's granulomatosis

- Tuberose sclerosis (lymphangioleiomyomatosis)

- Drug reactions, for example, amiodarone, methotrexate, bleomycin

\section{Rare lung diseases}

- Pulmonary alveolar proteinosis

- Pulmonary histiocytosis

- Pulmonary eosinophilia

- Idiopathic pulmonary haemosiderosis 
usual interstitial pneumonia (UIP), reticulation and honeycombing in a predominantly lower zone, sub-pleural distribution with minimal ground-glass opacities on HRCT, and relentlessly progressive breathlessness clinically, in the absence of known causes of fibrotic disease such as drugs, rheumatic diseases or environmental toxins or antigens. The characteristic histopathological features of UIP are a heterogeneous appearance with alternating areas of normal lung, interstitial inflammation, fibrosis, and honeycombing. ${ }^{56}$ The changes are most severe sub-pleurally and there is temporal heterogeneity such that the pathological processes are at different stages of development. The aetiology of IPF is quite unknown although there are associations with exposure to wood or metal dusts, cigarette smoking, viral infections, gastro-oesophageal reflux, and sometimes a family history of the disease. ${ }^{322-24}$ It may be the result of a failure of repair of lung tissue whereby injury culminates in fibrosis rather than a controlled inflammatory and healing process. ${ }^{72}$ The aberrant fibrosis may involve imbalances in the oxidantantioxidant and protease-antiprotease systems of the lung. IPF/UIP is a lethal disease with a $50 \%$ three year mortality. The mean age at presentation is 66 years, it is more common in men and in smokers, and clubbing is present in about $70 \%$ of patients. ${ }^{3}$ Response to treatment is poor. There is a lack of evidence from definitive trials but limited information from research studies and from consensus statements suggests that a combination of prednisolone $(0.5 \mathrm{mg} / \mathrm{kg} / \mathrm{day})$ and azathioprine $\left(2-3 \mathrm{mg} / \mathrm{kg} /\right.$ day) may be the best option. ${ }^{1526}$ There is now a tendency to use lower doses of prednisolone particularly in the maintenance phase to reduce adverse effects. A number of other treatments such as colchicine, penicillamine, interferon gamma, and pirfenidone have not proved effective. The antioxidant $\mathrm{N}$-acetylcysteine has been shown to slow the rate of decline in lung function but does not change mortality. ${ }^{27}$ Patients showing the expected progression of disease should be considered for lung transplantation if possible and for palliative care otherwise.

Acute interstitial pneumonia (AIP) is a very aggressive form of ILD that may occur as an acute phase of acceleration of IPF and is characterised by diffuse alveolar damage and the presence of hyaline membranes, similar to those found in the acute respiratory distress syndrome. ${ }^{2-5}$ Desquamative interstitial pneumonia (DIP) is a rare form of ILD that affects smokers and is characterised by desquamation of alveolar macrophages with minimal fibrosis. ${ }^{5}$ Respiratory bronchiolitis-ILD (RB-ILD) also occurs in smokers and is characterised by pigmented macrophages in the lumen of respiratory bronchioles with peri-bronchial fibrosis. ${ }^{28} 29$ DIP and RB-ILD respond well to corticosteroids, and smoking cessation is important. Lymphoid interstitial pneumonia (LIP) is characterised by the expansion of the interstitium by sheets of lymphoid cells. ${ }^{230}$ It may be idiopathic in nature or occur in association with connective tissue diseases, autoimmune diseases, or HIV infection, and in a small number of cases LIP undergoes transformation to lymphoma. ${ }^{30}$ Cryptogenic organising pneumonia (COP) is characterised by patchy intra-alveolar buds of organising fibrosis. It usually responds well to corticosteroids. ${ }^{51}$ Non-specific interstitial pneumonia (NSIP) is currently the second most common (after UIP) histopathological pattern of the idiopathic interstitial pneumonias and is characterised by a temporally and spatially uniform pattern that suggests that the lesions are of the same age. Fibroblastic foci and honeycombing are less prominent than in UIP and the response to corticosteroids is better. ${ }^{532}$

Physicians, pathologists, and radiologists are struggling to cope with the complexities of these diseases. It is important to appreciate that although the histopathological pattern is a key element in defining these diseases the overall diagnosis is not a pathological one as such, but entails integration of the clinical, radiological, and histopathological features. Thus the overall diagnosis is different depending on whether a particular histopathological pattern occurs without any known cause (idiopathic) or whether it occurs in association with a connective tissue disease, a drug reaction, or an environmental exposure to dust or antigens. ' The subclassification of the idiopathic interstitial pneumonias is also permitting more focused research into the pathogenesis of these diseases. Recently it has been shown that the genes expressed in lung tissue in IPF are those associated with tissue remodelling and myofibroblasts whereas the genes expressed in EAA are those associated with inflammation and $\mathrm{T}$ cell activation. ${ }^{25}$ The genes expressed in NSIP do not show a distinct pattern, suggesting that this may be a heterogeneous entity that requires further refinement. The stricter definition of IPF/UIP as a specific lethal disease and its distinction from less aggressive patterns of ILD are of crucial clinical importance.

\section{CONNECTIVE TISSUE DISEASES}

Pulmonary involvement is common in the connective tissue diseases and is not confined to parenchymal disease but spans the range of lung disease. ${ }^{33}$ Thus, for example, obstructive airways disease and bronchiectasis occur in rheumatoid disease, pleural effusions occur in systemic lupus erythematosus and rheumatoid disease, respiratory muscle weakness may complicate polymyositis, and pulmonary hypertension is an important cause of death in some forms of systemic sclerosis. All of the different histopathological patterns of ILD, such as UIP, NSIP, LIP, COP, occur in association with the connective tissue diseases and more than one pathology may be present in an individual patient. ${ }^{33}$ The prognosis and response to treatment is significantly better in UIP associated with a connective tissue disease than in UIP associated with IPF. HRCT or gas transfer measurements frequently detect sub-clinical ILD in patients with connective tissue disease, but this may be non-progressive and may not require treatment, although careful monitoring is needed. ILD may precede the systemic manifestations of a connective tissue disease and measurement of autoantibodies may be helpful. In systemic sclerosis Scl-70 antibodies are associated with ILD whereas anticentromere antibodies are associated with pulmonary vascular disease, and Jo-1 antibodies are associated with ILD in polymyositis. ${ }^{33-35}$

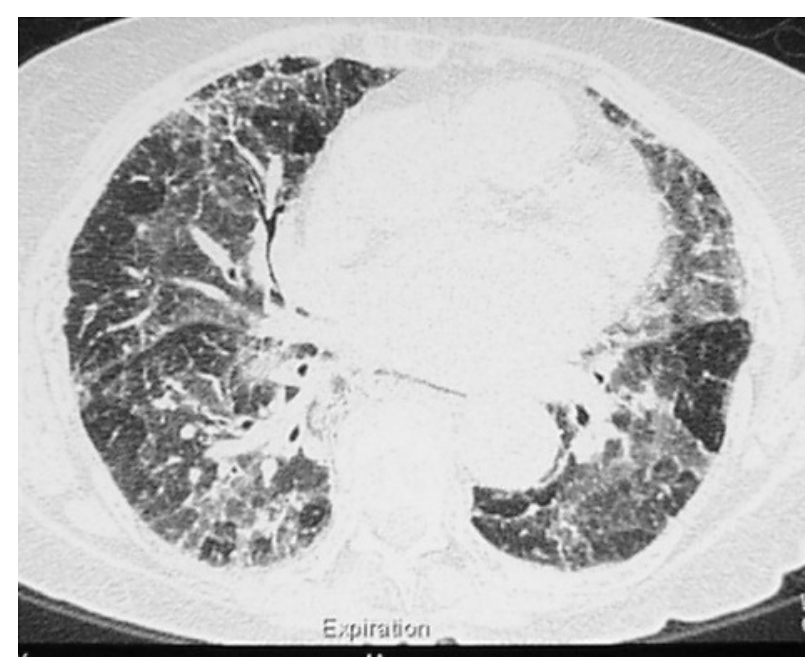

Figure 3 High resolution computed tomography showing ground-glass opacities and areas of decreased attenuation and air trapping in extrinsic allergic alveolitis. 
Many drug treatments (for example, methotrexate, lefluonamide) may cause ILD or induce immunosuppression and lung infection.

\section{DRUG INDUCED ILD}

Drug reactions are an important cause of ILD for physicians in many specialties. ${ }^{36}$ Amiodarone is a classic example of a drug that can produce an array of reactions within the lungs. ${ }^{36}$ Chest radiograph appearances include diffuse bilateral shadowing, asymmetrical infiltrates, and mass-like lesions. Lipid laden macrophages reflect amiodarone induced disturbance in phospholipid metabolism and may be associated with various histopathological features such as septal thickening, mononuclear cell infiltrates, fibrosis, and the patterns of NSIP, COP or UIP, for example. Often amiodarone lung has to be differentiated from pulmonary oedema, pulmonary infarction, infection, and other pathologies. A comparatively common problem is of a patient receiving anticancer chemotherapy (for example, bleomycin) who develops respiratory symptoms and lung infiltrates. ${ }^{36} 38$ Vigilance is required as new drugs are used in new circumstances. Thus for example, infliximab, a monoclonal antibody against tumour necrosis factor $\alpha$, is being used increasingly in treating patient with Crohn's disease or rheumatoid arthritis. Initially the most noticeable adverse effects were infections such as tuberculosis or pneumonia. However, a number of hypersensitivity lung reactions have recently been described including fibrosing alveolitis, eosinophilic pneumonia, and acute respiratory distress syndrome. ${ }^{39-43}$ In many of these circumstances the physician is faced with a patient with deteriorating respiratory disease that may be caused by infection, pulmonary involvement by the underlying disease, a drug reaction, or coincidental lung disease. The range of investigations for assessing diffuse parenchymal infiltrates needs to be applied judiciously in this situation and BAL is particularly helpful in detecting or excluding infection. Early recognition of drug induced ILD, prompt withdrawal of the drug, and corticosteroid treatment are important. Up to date information is available on a dedicated web site (http://www.pneumotox.com).

\section{EXTRINSIC ALLERGIC ALVEOLITIS}

EAA is an immunologically mediated disease resulting in a hypersensitivity pneumonitis with $\mathrm{T}$ cell lymphocytic infiltration and granulomatous inflammation in the bronchioles and alveoli, from the repeated inhalation of certain antigens in a sensitised person..$^{184} 45$ The commonest types of EAA are bird fancier's lung, typically in relation to a pet budgie or the sport of pigeon racing, farmer's lung attributable to fungal spores in mouldy hay, and humidifier lung attributable to contamination of various water systems in the home or workplace. In the acute form the patient experiences recurrent episodes of dyspnoea, cough, fever, and a flu-like sensation and the chest radiograph typically shows alveolar shadowing. The key to diagnosis is the identification of exposure to a provoking antigen and a corresponding antibody response with typical clinical features. The diagnosis of acute EAA can usually be made on the basis of clinical features and simple investigations, and lung biopsy is rarely required. ${ }^{17}$ HRCT typically shows ground-glass opacities with areas of decreased attenuation on expiratory images because of air trapping and bronchiolitis (fig 3). The chronic form of EAA is characterised by the insidious development of dyspnoea and lung fibrosis, which may be difficult to differentiate from other fibrotic lung diseases. Lung biopsy at this stage of EAA may show patterns similar to NSIP, UIP, and COP. ${ }^{46}{ }^{47}$ Although ILD is the characteristic outcome of EAA, chronic bronchitis, airways obstruction, and emphysema also occur. ${ }^{48}{ }^{49}$ Withdrawal from antigen exposure is

\section{Key references}

- American Thoracic Society/European Respiratory Society. International multidisciplinary classification of the idiopathic interstitial pneumonias. Am J Respir Crit Care Med 2002;165:277-304.

- King TE. Clinical advances in the diagnosis and therapy of the interstitial lung diseases. Am J Respir Crit Care Med 2005; 172:268-79.

- Lacasse Y, Selman M, Costabel U, et al. Clinical diagnosis of hypersensitivity pneumonitis. Am J Respir Crit Care Med 2003;168:952-8.

- Martin WJ, lannuzzi MC, Gail DP, et al. Future directions in sarcoidosis research. Am J Respir Crit Care Med 2004; 130:567-71.

the mainstay of treatment. Corticosteroids hasten the resolution of acute EAA but do not change the ultimate level of lung function..$^{50}$

\section{SARCOIDOSIS}

Sarcoidosis remains a mysterious disease, characterised by the occurrence in affected organs of granulomatous lesions that may progress to fibrosis. Because of its diverse manifestations it may present to clinicians in many specialties. $^{5152}$ The accumulation of CD4 lymphocytes at disease sites, particularly the lungs, suggests an immunological reaction to an unidentified inhaled antigen in a genetically susceptible person. The search for a sarcoid antigen has been frustratingly unrewarding, but progress has been made in understanding the clinical behaviour of the disease.

The clinical features of sarcoidosis are varied but at either end of the range are an acute form, characterised by bilateral hilar lymphadenopathy, erythema nodosum, and uveitis (Loefgren's syndrome) and chronic sarcoidosis, which may affect virtually any organ in the body. Central nervous system involvement may give rise to cranial neuropathies, chronic meningitis, and hydrocephalus. ${ }^{53}$ Cardiac involvement may cause conduction system damage and arrhythmias, and corticosteroid therapy is usually started immediately in such cases. Diagnosis is based on histological evidence of noncaseating granulomas in association with typical clinical features, in the absence of other causes of granulomatous disease. Bronchoscopy with BAL and transbronchial biopsy is often diagnostic. Investigations depend on the clinical context and mediastinoscopy with lymph node biopsy is often required to confirm sarcoidosis and to exclude tuberculosis or lymphoma. A patient presenting with classic Loefgren's syndrome may not require biopsy. Once the diagnosis is made it is then necessary to assess the extent and severity of the disease. The location of sarcoid lesions is crucial because even limited granulomatous inflammation of

\section{Useful web sites}

- British Thoracic Society http://www.brit-thoracic. org.uk

- American Thoracic Society http://www.thoracic.org

- British Lung Foundation http://www.lunguk.org

- Arthritis Research Campaign http://www.arc.org.uk

- Pneumotox drug reactions http://www.pneumotox. com 
the heart or nervous system may have important consequences, whereas extensive lymph node involvement may have little importance. Often a period of observation is appropriate to assess whether the disease resolves spontaneously or progresses. The factors determining progression are poorly understood although genetic and ethnic factors, relating to some HLA phenotypes, seem to be important in determining the pattern of disease and progression. ${ }^{51} 52$ Previous research concepts of disease activity and inflammatory levels using serum angiotensin converting enzyme measurement, BAL lymphocyte counts, and gallium scans, have not proved useful clinically. Thus patients with the highest lymphocyte counts on BAL are those with Loefgren's syndrome yet the disease in these patients is most likely to resolve spontaneously. ${ }^{54}$ The level of inflammation does not predict progression to fibrosis. Most patients recover spontaneously and the overall prognosis is excellent but about $10 \%$ sustain permanent damage in affected organs and $1 \%-5 \%$ of patients referred to specialist centres die from complications such as respiratory failure or neurological or cardiac involvement. Studies of the effect of corticosteroids on the long term outcome of sarcoidosis have been difficult because of the very variable course of the disease. However the British Thoracic Society study showed an improved clinical outcome, at the cost of some side effects, in patients treated with corticosteroids after they had failed to show spontaneous improvement over a six month observation period..$^{55}$

Progress has been made in recognising the different patterns of ILD and this has led to a better understanding of the various clinical syndromes. Problems persist because of the inherent complexity of these diseases and the limited progress in understanding their pathogenesis. The multidisciplinary approach to ILD by physicians, radiologists, and pathologists is proving promising.

\section{QUESTIONS (ANSWERS AT THE END OF THE REFERENCES)}

Choose the best of the five options for each question.

1. The worst clinical prognosis is associated with the histopathological pattern of:

(A) non-specific interstitial pneumonia

(B) desquamative interstitial pneumonia

(C) cryptogenic organising pneumonia

(D) usual interstitial pneumonia

(E) lymphoid interstitial pneumonia

2. Bronchoalveolar lavage in sarcoidosis typically has a high count of:

(A) lymphocytes

(B) eosinophils

(C) haemosiderin laden macrophages

(D) neutrophils

(E) histiocytes

3. Pulmonary hypertension in systemic sclerosis is associated with:

(A) Scl-70 antibodies

(B) an increased serum angiotensin converting enzyme activity

(C) anti-Jo antibodies

(D) antismooth muscle antibodies

(E) anticentromere antibodides
4. The most characteristic feature of idiopathic pulmonary fibrosis on computed tomography is:

(A) honeycombing

(B) pleural thickening

(C) ground-glass opacities

(D) areas of decreased attenuation

(E) peri-hilar reticular shadow

5. The clinical features of clubbing, crackles and progressive dyspnoea are most suggestive of a diagnosis of:

(A) acute interstitial pneumonia

(B) idiopathic interstitial pneumonia

(C) idiopathic pulmonary fibrosis

(D) extrinsic allergic alveolitis

(E) cryptogenic organising pneumonia

Funding: none.

Conflicts of interest: none.

\section{REFERENCES}

1 Cushley MJ, Davison AG, DuBois RM, et al. The diagnosis, assessment and treatment of diffuse parenchymal lung disease in adults: British Thoracic Society recommendations. Thorax, 1999;54(suppl 1).

2 King TE. Clinical advances in the diagnosis and therapy of interstitial lung diseases. Am J Respir Crit Care Med 2005;172:268-79.

3 DuBois TM, Costabel U. Diffuse lung disease: classification and diagnostic approach. In: Gibson GJ, Geddes DM, Costabel U, et al. Respiratory medicine. London: Elsevier Science, 2003:1545-56.

4 Johnson IDA, Prescott RJ, Chalmers JC, et al. British Thoracic Society study of cryptogenic fibrosing alveolitis: current presentation and initial management. Thorax 1997; 52:38-44.

5 Joint statement of the American Thoracic Society and European Respiratory Society. Idiopathic pulmonary fibrosis: diagnosis and treatment. Am J Respir Crit Care Med 2000;161:646-64.

6 American Thoracic Society/European Respiratory Society. International multidisciplinary classification of the idiopathic interstitial pneumonias. Am J Respir Crit Care Med 2002; 165:277-304.

7 Ward PA, Hunninghake GW. Lung inflammation and fibrosis. Am J Resp Crit Care Med 1998;157:s123-9.

8 Kennedy Jl, Myers JL, Plumb VJ, et al. Amiodarone pulmonary toxicity: clinical, radiologic and pathologic correlations. Arch Intern Med 1987; 147:50-5.

9 Flaherty KR, King TE, Raghu G, et al. Idiopathic interstitial pneumonia: what is the effect of a multidisciplinary approach to diagnosis? Am J Respir Crit Care Med 2004; 170:904-10.

10 McLoud T. Role of high-resolution computed tomography in idiopathic pulmonary fibrosis. Am J Respir Crit Care Med 2005;172:408-9.

11 Lynch DA, Godwin JD, Safrin S, et al. High-resolution computed tomography in idiopathic pulmonary fibrosis. Am J Respir Crit Care 2005;172:488-93.

12 Drent M, Jacobs JA, Wagenaar SS. Bronchoalveolar lavage. Eur Respir Mon 2000; 14:63-78.

13 Langford CA, Hoffman GS. Wegener's granulomatosis. Thorax 1999;54:629-37.

14 Vasallo R, Ryu IH, Colby TV, et al. Pulmonary Langerhans cell histiocytosis. N Engl J Med 2000;342:1969-77.

15 Seymour JF, Presneill JJ. Pulmonary alveolar proteinosis. Am J Respir Crit Care Med 2002;166:215-35.

16 Fishbein $M$. To biopsy or not biopsy: assessing the role of surgical biopsy in the diagnosis of idiopathic pulmonary fibrosis. Chest 2005; 128:s520-5.

17 Lacasse Y, Selman M, Costabel U, et al. Clinical diagnosis of hypersensitivity pneumonitis. Am J Respir Crit Care Med 2003; 168:952-8.

18 Bourke SJ, Dalphin JC, Boyd G, et al. Hypersensitivity pneumonitis: current concepts. Eur Respir J 2001;18(suppl 32):81-92.

19 British Thoracic Society. Guidelines on diagnostic flexible bronchoscopy. Thorax 2001;56(suppl 1):1-21.

20 Lettieri CJ, Veerappan GR, Helman DL, et al. Outcomes and safety of surgical lung biopsy for interstitial lung disease. Chest 2005; 127:1600-5.

21 Wells AU. Histopathological diagnosis in diffuse lung disease: an ailing gold standard. Am J Respir Crit Care Med 2004;170:828-9.

22 Hubbard R, Lewis S, Richards K, et al. Occupational exposure to metal or wood dust and aetiology of cryptogenic fibrosing alveolitis. Lancet 1996;347:284-9.

23 Raghu G, Freudenberger TD, Yang S, et al. High prevalence of abnormal acid grastroesophageal reflux in idiopathic pulmonary fibrosis. Eur Respir $J$ 2006;27:136-42.

24 Steele MP, Speer MC, Loyd JE, et al. Clinical and pathologic features of familial interstitial pneumonia. Am J Respir Crit Care Med 
25 Selman M, Pardo A, Barrera L, et al. Gene expression profiles distinguish idiopathic pulmonary fibrosis from hypersensitivity pneumonitis. Am J Respir Crit Care Med 2006;173:188-98.

26 Bouros D, Antoniou KM. Current and future therapeutic approaches in idiopathic pulmonary fibrosis. Eur Respir J 2005;26:693-702.

27 Dermedts $M$, Behr J, Buhl R, et al. High-dose acetylcysteine in idiopathic pulmonary fibrosis. N Engl J Med 2005;353:2229-42.

28 Myers JL, Veal CF, Shin MS, et al. Respiratory bronchioloitis causing interstitial lung disease: a clinicopathological study of six cases. Am Rev Respir Dis 1987;135:880-4.

29 Mavridou D, Laws D. Respiratory bronchiolitis associated interstitial lung disease (RB-ILD): a case of an acute presentation. Thorax 2004;59:910-11.

30 Swigris JJ, Berry GJ, Raffin TA, et al. Lymphoid interstitial pneumonia. Chest 2002;122:2150-64.

31 Bellomo R, Finlay M, McLaughlin P, et al. Clinical spectrum of cryptogenic organising pneumonitis. Thorax 1991;46:554-8.

32 Riha RL, Duhig EE, Clarke BE, et al. Survival of patients with biopsy-proven usual interstitial pneumonia and non-specific interstitial pneumonia. Eur Respir J 2002;19:1114-18.

33 Wells AU. Lung disease in association with connective tissue diseases. Eur Respir Mon 2000;14:137-64.

34 Douglas WW, Tazelaar HD, Hartman TE, et al. Polymyositis-dermatomyositisassociated interstitial lung disease. Am J Respir Crit Care Med 2001;164:1182-5.

35 Owens GR, Fallansbee WP. Cardiopulmonary manifestations of systemic sclerosis. Chest 1987;91:118-27.

36 Camus P, Gibson GJ. latrogenic respiratory disease. In: Gibson GJ, Geddes DM, Costabel U, et al. Respiratory medicine. London: Elsevier Science, 2003:764-806.

37 Adams PC, Gibson GJ, Morley AR, et al. Amiodarone pulmonary toxicity: clinical and subclinical features. Q J Med 1986;59:449-71.

38 White DA, Stover DE. Severe bleomycin-induced pneumonitis: clinical features and response to corticosteroids. Chest 1984:86:723-8.

39 British Thoracic Society. Recommendations for assessing risk and for managing Mycobacterium tuberculosis infection and disease in patients due to start anti-TNF-alpha treatment. Thorax 2005;60:800-5.

40 Colombel JF, Loftus EV, Tremaine WJ, et al. The safety profile of infliximab in patients with Crohn's disease: the Mayo Clinic experience in 500 patients. Gastroenterology 2004;126: 1-23.

41 Ostor AJ, Crisp AJ, Somerville MF, et al. Fatal exacerbation of rheumatoid arthritis associated fibrosing alveolitis in patients given infliximab. $B M J$ 2004;329:1266-8.
42 Riegert-Johnson DL, Godfrey JA, Myers JL, et al. Delayed hypersensitivity reaction and acute respiratory distress syndrome following infliximab infusion. Inflammatory Bowel Disease 2002;8:186-91.

43 Ledingham J, Deighton C. Update on the British Society for Rheumatology guidelines for prescribing TNF alpha blockers in adults with rheumatoid arthritis. Rheumatology 2005;44:157-63.

44 Bourke SJ, Boyd G. Pigeon fancier's lung. BMJ 1997;315:70-1.

45 Nemery B, Bast A, Behr J, et al. Interstitial lung disease induced by exogenous agents: factors governing susceptibility. Eur Respir J 2001;18(suppl 32):30-42.

46 Ohtani Y, Saiki S, Kitaichi M, et al. Chronic bird fancier's lung: histopathological and clinical correlation. An application of the 2002 ATS/ ERS consensus classification of the idiopathic interstitial pneumonias. Thorax 2005;60:665-71

47 Perez-Padilla R, Salas J, Chapela R, et al. Mortality in Mexican patients with chronic pigeon breeder's lung compared with those with usual interstitial pneumonia. Am Rev Respir Dis 1993;148:49-53.

48 Bourke SJ, Anderson K, Lynch P, et al. Chronic simple bronchitis in pigeon fancier's. Chest 1989;95:598-601.

49 Bourke SJ, Carter R, Anderson K, et al. Obstructive airways disease in nonsmoking subjects with pigeon fancier's lung. Clin Expt Allergy 1989; 19:629-32

50 Kokkarinen Jl, Tukiainen HO, Terho EO. Effect of corticosteroid treatment on the recovery of pulmonary function in farmer's lung. Am Rev Respir Dis 1992; 145:3-5.

51 American Thoracic Society. Statement on sarcoidosis. Am J Respir Crit Care Med 1999; 160:736-55.

52 Martin WJ, lannuzzi MC, Gail DB, et al. Future directions in sarcoidosis research. Am J Respir Crit Care Med 2004;170:567-71.

53 Zajicek JP, Scolding NJ, Foster O, et al. Central nervous system sarcoidosisdiagnosis and management. Q J Med 1999:91:103-17.

54 Ward K, O'Connor C, Odlum C, et al. Prognostic value of bronchoalveolar lavage in sarcoidosis: the critical influence of disease presentation. Thorax 1989;44:6-12.

55 Gibson GJ, Prescott RJ, Muers MF, et al. British Thoracic Society sarcoidosis study: effects of long term corticosteroid treatment. Thorax 1996;51:238-47.

\section{ANSWERS}

1. (D); 2. (A); 3. (E); 4. (A); 5. (C). 